\title{
Journal of Clinical \&. Experimental Ophthalmology
}
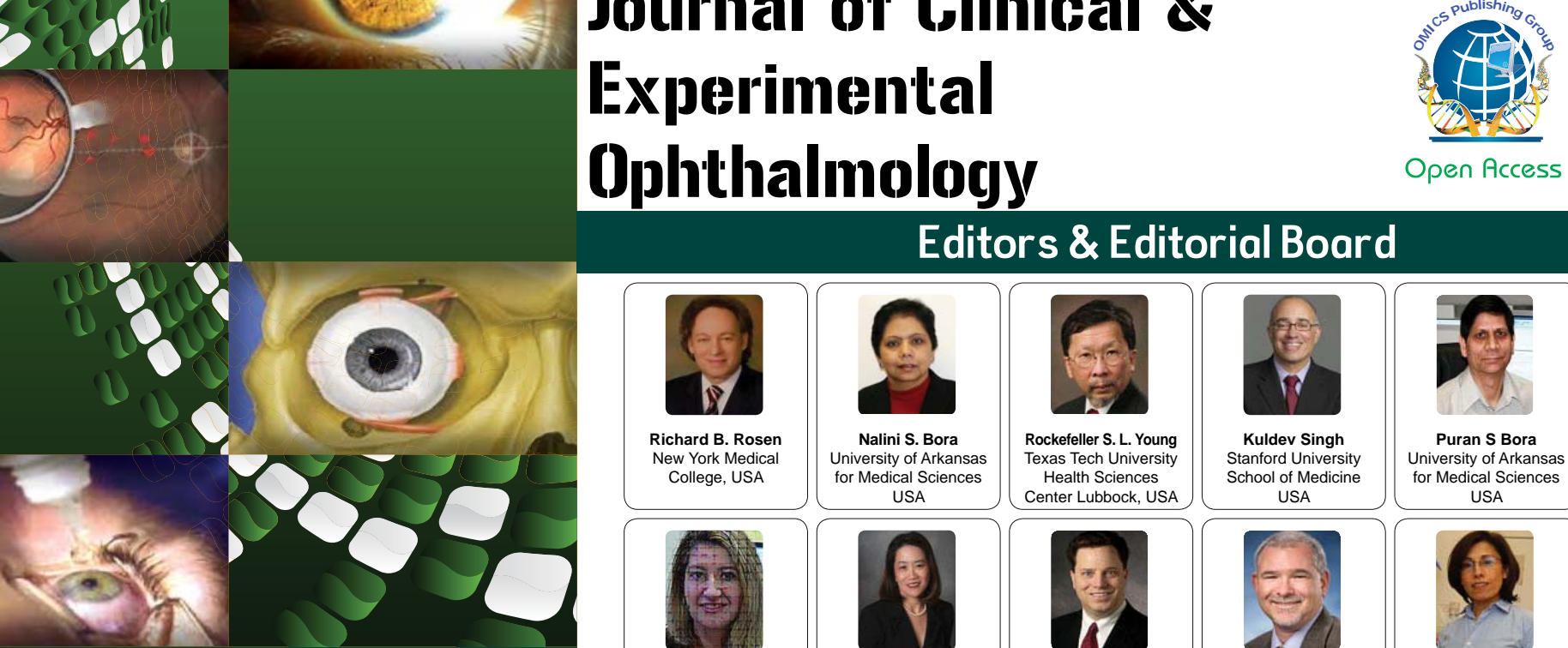

phthalmology is a medical
specialty dealing with the study, treatment, function and diseases of the eye. The study elaborates the surgical treatment of eye disorders ensures the care and prevention of blindness. The Journal of Clinical and Experimental Ophthalmology is a peer-reviewed ophthalmic journal; international in its scope not only renders ophthalmic studies and research advancements in surgical treatments. This journal is reliable and is freely accessible through net globally to share the innovations and advancements of ophthalmologists

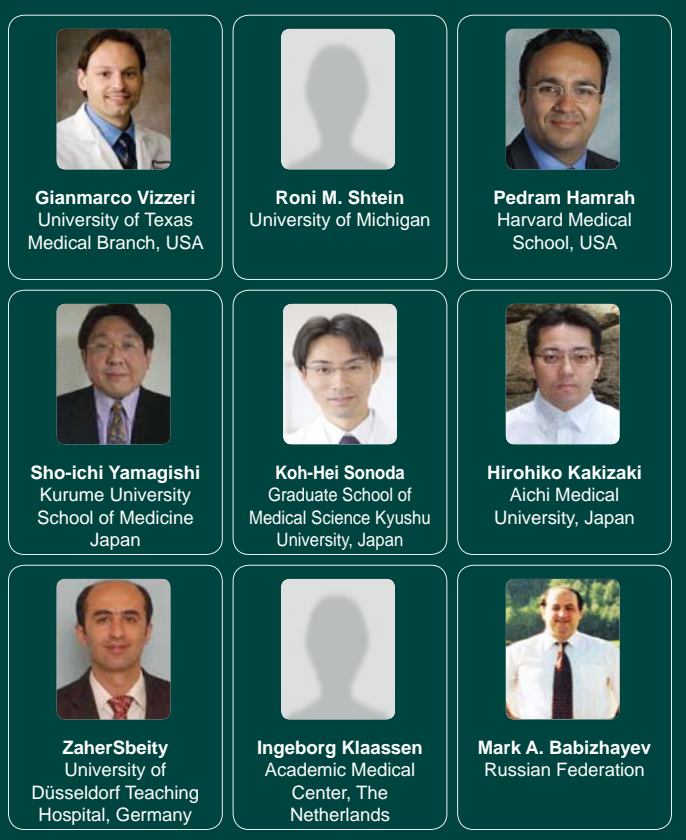

OMICS Publishing Group

5716 Corsa Ave., Suite 110, Westlake, Los Angeles, CA

91362-7354, USA, Phone: +1- 650-268-9744

Fax: +1-650-618-1414, Toll free: +1-800-216-6499

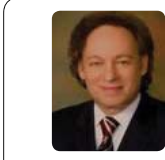
New York Medical College, USA

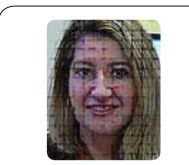

Ekaterini Tsilou

for Mothers and

Children, USA

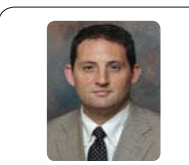

Stephen G Schwartz Bascom Palmer Eye Institute at Naples
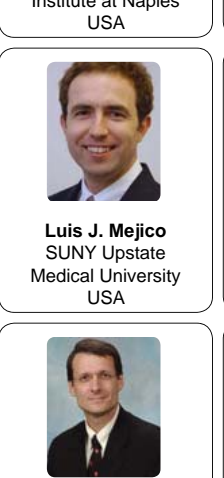

Michael w Stewart Mayo Medicine, USA
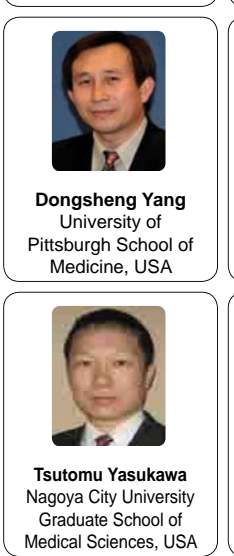

Medical Sciences, USA

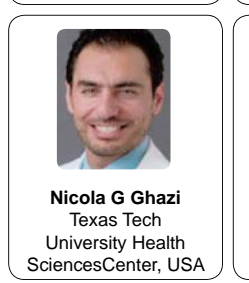

Editors \& Editorial Board
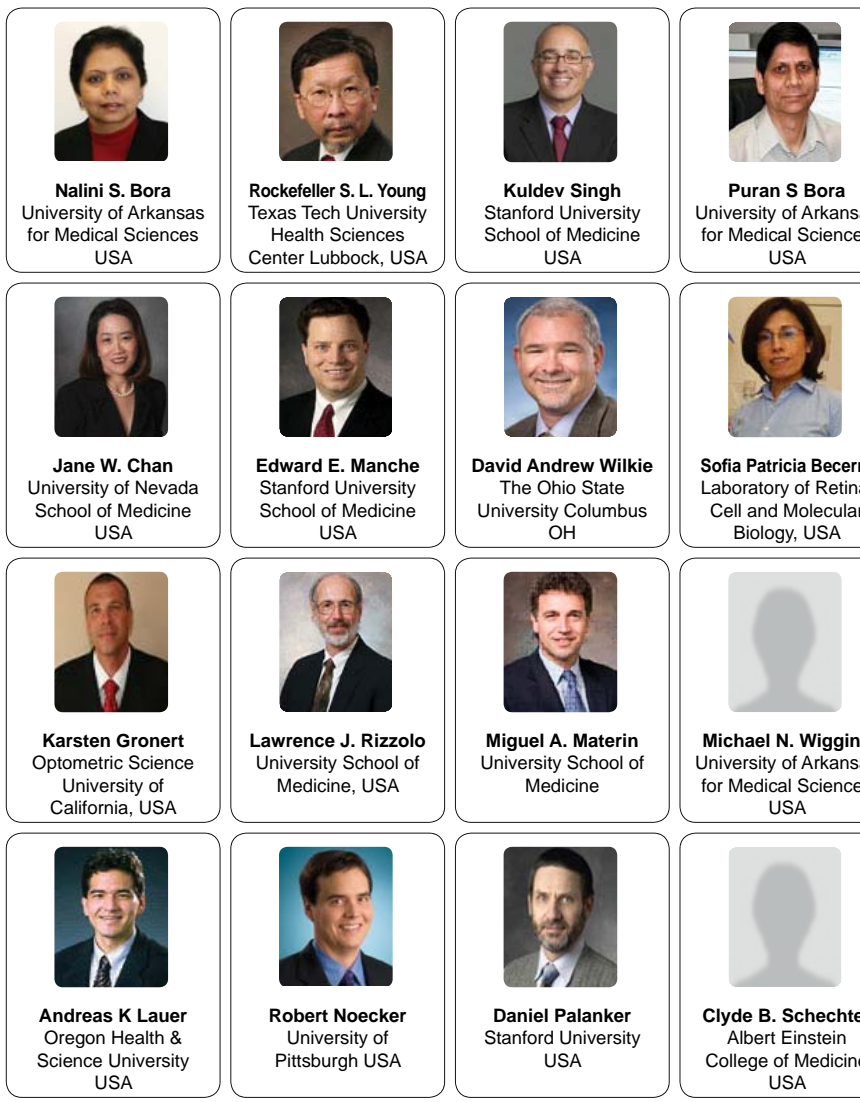

Kuldev Singh Stanford University

School of Medicin

Puran S Bora University of Arkansas University of Arkansas
for Medical Sciences
USA Center Lubbock, USA

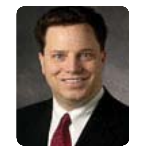

Edward E. Manche Stanford University

School of Medicine
USA

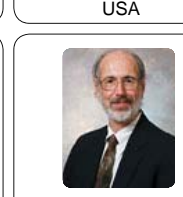

Lawrence J. Rizzolo University School of Medicine, USA

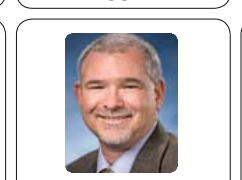

David Andrew Wilkie The Ohio State University Columbus
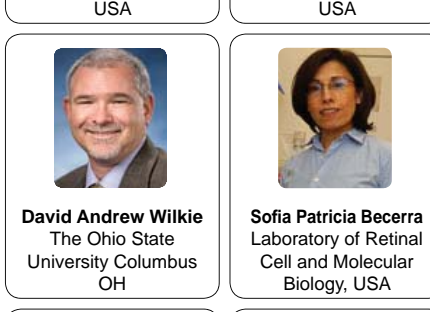

Sofia Patricia Becerra Laboratory of Retinal Laboratory of Retinal Cell and Molecular
Biology, USA

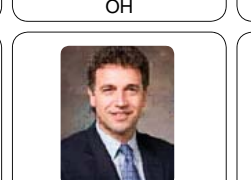

\section{Miguel A. Materin} Medicine

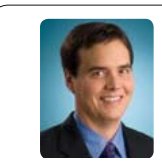

Robert Noecker University of Pittsburgh USA
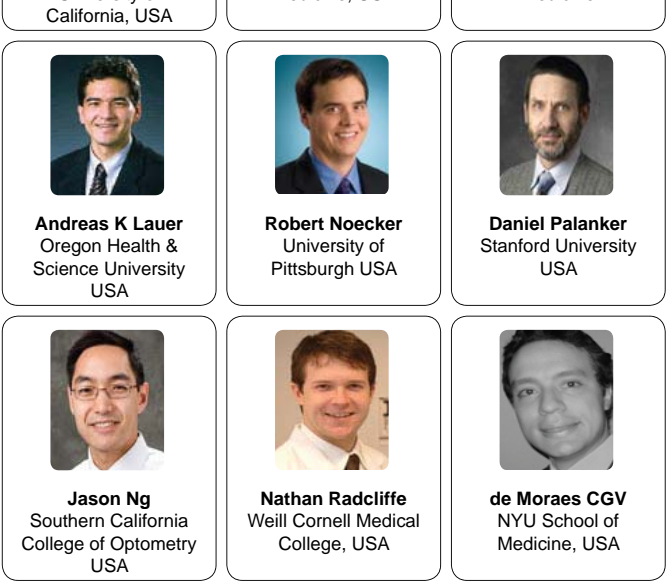

Michael N. Wiggins for Medical Sciences for Medical Scie
USA
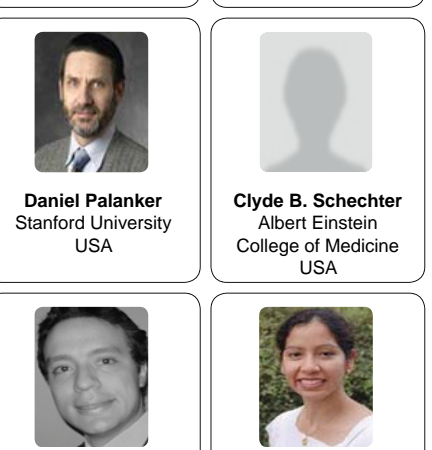

de Moraes CGV NYU School of Medicine, USA
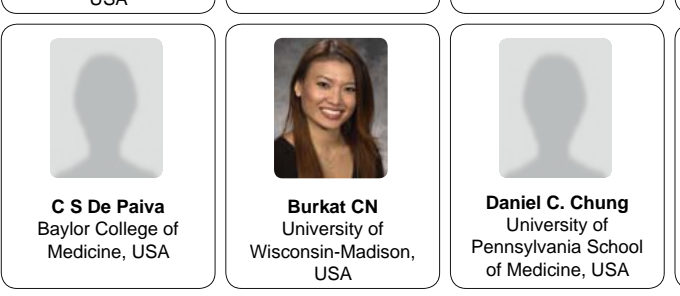

Gabriela Espinoza Saint Louis University Eye Institute, USA

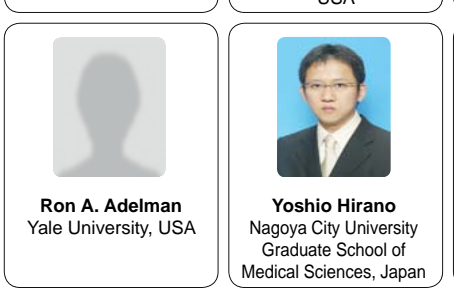

of Medicine, USA

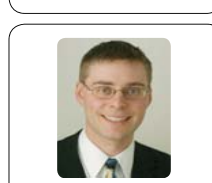

Aljoscha S. Neubauer Ludwig Maximilians University, USA
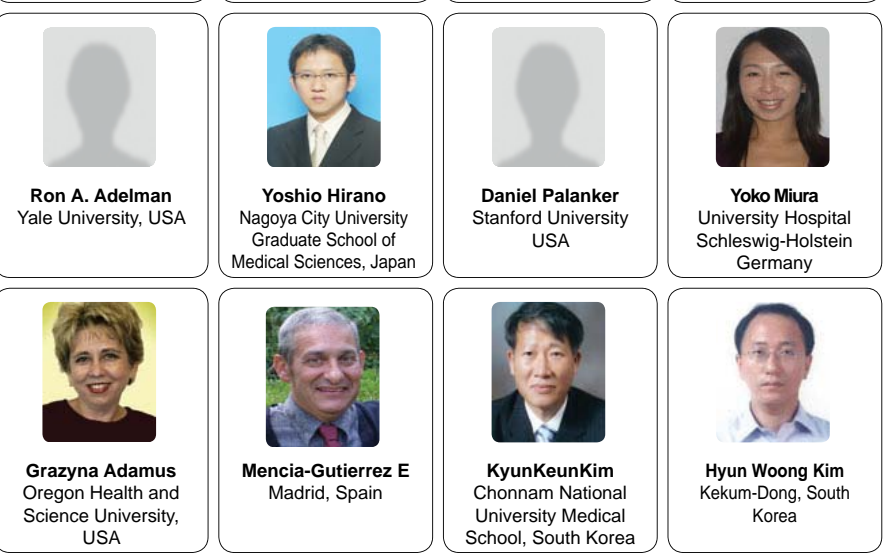

http://www.omicsonline.org/jceohome.php 


\title{
Subretinal Lavage to Prevent Persistent Subretinal Fluid after Rhegmatogenous Retinal Detachment Surgery: A Study of Feasibility and Safety
}

\author{
Marc Veckeneer*, Elisabeth van Aken, David Wong, Rudolf Reyniers and Jan van Meurs
}

Rotterdam Eye Hospital, Schiedamsevest 180, 3011 BH Rotterdam, The Netherlands

\begin{abstract}
Purpose: To report the proof of concept of a surgical innovation.

Background: Optical coherence tomography often identifies persistent subretinal fluid (PSF) after apparently successful retinal detachment repair surgery. Based on the literature, we hypothesised that highly viscous PSF, which interferes with the normal function of the retinal pigment epithelium, can lead to these persistent blebs. We therefore devised a novel surgical manoeuvre of subretinal lavage to dilute the subretinal fluid (SRF) during surgery. We expected that this would reduce the incidence of PSF.
\end{abstract}

Methods: We report our experience with a modified surgical drainage technique carried out in 12 eyes of 12 patients with long standing retinal detachments. We implemented subretinal lavage combined with vitrectomy and gas.

Results: None of the patients developed PSF.

Conclusion: We concluded that the novel technique of subretinal lavage was safe and feasible. We propose that a controlled trial would be worthwhile.

Keywords: Persistent submacular fluid; Rhegmatogenous retinal detachment

Abbreviations: PSF: Persistent Submacular Fluid; SRF: Subretinal Fluid; RRD: Rhegmatogenous Retinal Detachment; OCT: Optical Coherence Tomography; MV: Marc Veckeneer

\section{Introduction}

After surgery for rhegmatogenous retinal detachment (RRD), although the retina appears fully attached by ophthalmoscopy, some subretinal fluid (SRF) may persist. The introduction of optical coherence tomography (OCT) resulted in multiple reports of persistent subretinal fluid (PSF) after RRD surgery [1-4].

It is currently difficult to link the incidence of PSF to any particular clinical characteristics. Both Wolfensberger and Benson et al. reported that PSF occurred more frequently after buckling than after vitrectomy [5-7]. They suggested that scleral buckling might disturb the choroidal circulation and, consequently, impair SRF absorption [8]. Others suggested that cryotherapy might break down the blood-ocular barrier. However, this seems unlikely, because various vitrectomy cases used cryotherapy and reported no blebs; but, in contrast, we avoided cryotherapy in scleral buckling surgery and, nevertheless, observed PSF in several cases. Finally, it is possible that intraocular gas bubbles might displace any SRF away from the macula; however, neither Wolfensberger nor Benson found any relationship between postoperative posturing and incidence of PSF.

We generated a new hypotheses based on the observation that PSF tended to occur in patients with long-standing retinal detachment and viscous SRF. Upon examining these SRF samples by immunohistochemistry and electron microscopy, we found high levels of cellular debris, particularly photoreceptor outer segments [9]. We therefore hypothesised that the viscous nature of the SRF interfered with its spontaneous, complete absorption by the retinal pigment epithelium, and this could lead to postoperative blebs. The aim of this interventional study was to test this hypothesis by determining whether subretinal lavage could prevent persistent subretinal blebs, and to establish the feasibility and safety of this novel technique.

\section{Materials and Methods}

This study included non-consecutive patients with primary macula-off RRD that were scheduled for vitrectomy surgery. Selection criteria included cases of RRD that arose from retinal breaks situated away from the deepest SRF, with estimated retinal detachment (RD) duration of at least 1 week. In these cases, SRF can be highly viscous and impossible to drain completely through the existing breaks. Rather than creating an extra retinal break near the posterior pole to complete the drainage of residual fluid, transscleral drainage was performed. Patients with previous ocular history, including cataract surgery, were excluded. This study was approved by the Institutional Research Board of the Rotterdam Eye Hospital. In accordance with the IDEAL recommendations on the stages of development of surgical innovation [10], this study was defined as stage 1. Although the combination of surgical techniques was not reported before, we had previous experience with this approach. No new instruments or pharmacological aids of an experimental nature were used. All surgical procedures were performed by one surgeon (MV).

All patients underwent full ophthalmologic assessment,

*Corresponding author: Marc Veckeneer, Rotterdam Eye Hospital Schiedamsevest 180, 3011 BH Rotterdam, The Netherlands, Tel: 31104017642 Fax: 31104111747; E-mail: veckeneer.icare@gmail.com

Received February 23, 2011; Accepted April 05, 2011; Published April 09, 2011

Citation: Veckeneer M, van Aken E, Wong D, Reyniers R, van Meurs J (2011) Subretinal Lavage to Prevent Persistent Subretinal Fluid after Rhegmatogenous Retinal Detachment Surgery: A Study of Feasibility and Safety. J Clinic Experiment Ophthalmol 2:154. doi:10.4172/2155-9570.1000154

Copyright: ( 2011 Veckeneer M, et al. This is an open-access article distributed under the terms of the Creative Commons Attribution License, which permits unrestricted use, distribution, and reproduction in any medium, provided the original author and source are credited. 
Citation: Veckeneer M, van Aken E, Wong D, Reyniers R, van Meurs J (2011) Subretinal Lavage to Prevent Persistent Subretinal Fluid after Rhegmatogenous Retinal Detachment Surgery: A Study of Feasibility and Safety. J Clinic Experiment Ophthalmol 2:154. doi:10.4172/21559570.1000154

Page 2 of 3

preoperatively and 4 weeks after surgery. The assessment included bestcorrected visual acuity, anterior segment examination, fundoscopy with indirect ophthalmoscopy, and slit-lamp biomicroscopy with a Volk Super Quad contact lens. The extent of RD was expressed as the number of retinal quadrants that showed detachment. At the 4 -week postoperative visit, we performed a Stratus OCT scan with a $6 \times 6 \mathrm{~mm}$ radial line scan protocol.

The surgical procedure comprised a 23-gauge 3-port pars plana vitrectomy. Bimanual manipulation was facilitated by chandelier illumination. Rather than flattening the retina with perfluorocarbon liquids, we performed modified transscleral drainage. Briefly, a 27 gauge needle, fitted with an insertion tool (normally used for fluid drainage during conventional sclera buckling surgery) (Figure 1), was connected by tubing to a 3 -way stopcock with two $5 \mathrm{cc}$ syringes, one filled with balanced salt solution. The needle was introduced into the subretinal space in the area of highest retinal elevation (Figure 2). The subretinal space was then inflated with BSS in order to dilute the viscous SRF. The intra-ocular pressure was normalised by venting vitreous fluid via a flute needle positioned in the mid-vitreous cavity (Figure 3). Then, after turning the stopcock, SRF was aspirated into the second syringe; this resulted in partial flattening of the RRD. At least 2 cycles of inflation and aspiration were performed. Finally, the needle was removed from the subretinal space as the retina re-attached itself. The evacuation of

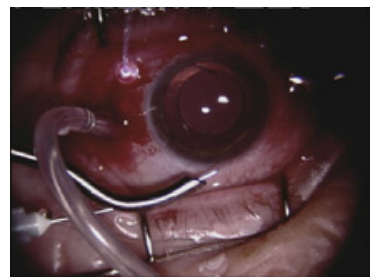

Figure 1: The insertion tool for introduction of the needle during transscleral drainage is the same as used during buckling surgery.

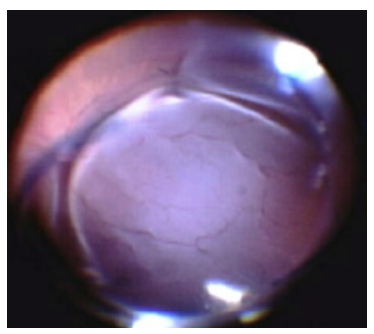

Figure 2: Lavage is performed through a 27 gauge needle inserted transsclerally into the subretinal space in the area of highest retinal elevation.

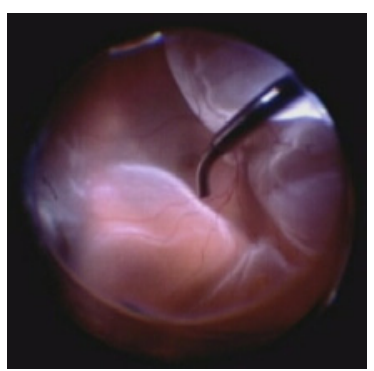

Figure 3: Maintaining stable intraocular pressure by venting vitreous fluid via a flute needle positioned in the mid-vitreous cavity.

\begin{tabular}{|l|l|}
\hline Age (yrs) & $68(54-80)$ \\
\hline Gender (female, male) & 3,9 \\
\hline Eye (right, left) & 10,2 \\
\hline Duration of detachment (days) & $12(7-60)$ \\
\hline $\begin{array}{l}\text { Type of detachment } \\
\text { (round hole, horseshoe tear) }\end{array}$ & 3,9 \\
\hline Number of breaks & $1(1-3)$ \\
\hline Clock hours detachment & $6(4-12)$ \\
\hline BCVA, pre-op & $0.033(0.003-0.16)$ \\
\hline BCVA, post-op & $0.16(0.05-0.6)$ \\
\hline Follow up (wks) & $14(6-28)$ \\
\hline
\end{tabular}

Table 1: Demographic data and outcome of 12 Patients.

any residual peripheral SRF was accelerated by raising the infusion bottle to increase the hydrostatic pressure in the vitreous cavity. For the surgeon not accustomed to transscleral drainage, alternatively, diluting the subretinal fluid could be performed by infusing fluid through a dual bore needle inserted into an existing break. The procedure is demonstrated in Video 1 . The vitreous base was shaved as previously described [11].

\section{Results}

Twelve patients were recruited from March through July 2008. Demographic data and functional outcome are shown in Table 1. In two cases, minor subretinal haemorrhages (not clinically elevated) occurred at the transscleral needle introduction site. The procedure was otherwise straightforward and uncomplicated in all cases. Four weeks after surgery, ophthalmoscopy showed re-attached retinas in all twelve patients. No patients exhibited subretinal blebs or subclinical detachment on the OCT.

The values are the median(range) unless otherwise stated; BCVA: best corrected visual acuity, as tested on the Snellen chart (logMar converted); post-op: 4 weeks after surgery.

\section{Discussion}

Delayed or incomplete visual recovery after RD surgery remains an important problem involving many factors $[12,13]$. With OCTfacilitated diagnostics, PSF has become recognised as an additional factor. Although some visual improvement typically occurs with the disappearance of PSF, spontaneous resolution can take many months $[4,6,7,16]$. It is unknown whether a better outcome might be achieved by rapid fluid absorption. However, longstanding fluid between the RPE and photoreceptor layer whether it be hyaluronic acid as in experimental RD or fluid originating from the choriocapillaris as in chronic central serous chorioretinopathy causes the photoreceptor layer to progressively atrophy over time [17].

In fresh detachments, when all retinal breaks are closed, an oncotic pressure gradient and an active retinal pigment epithelium pump favour rapid fluid absorption [18]. Similarly, after a fresh bullous detachment, pneumatic retinopexy can result in complete re-attachment within 24 hours.

Longstanding detachments have a different pathophysiology. They frequently occur in phakic eyes with small breaks, particularly at inferior locations $[2,19]$. Non-syneretic vitreous may slow the progression of RD by tamponading the retinal breaks. Similarly, these characteristics of "young" vitreous may play a role in PSF. The highly viscous, proteinrich SRF may show enhanced adherence to retinal pigment epithelium after the excess SRF is drained.

Interestingly, the oncotic pressure in SRF increases with the 
Citation: Veckeneer M, van Aken E, Wong D, Reyniers R, van Meurs J (2011) Subretinal Lavage to Prevent Persistent Subretinal Fluid after Rhegmatogenous Retinal Detachment Surgery: A Study of Feasibility and Safety. J Clinic Experiment Ophthalmol 2:154. doi:10.4172/21559570.1000154

duration of detachment $[20,21]$. After detachment, the loss of microvilli and the presence of hyaluronic acid can inhibit the ability of the retinal pigment epithelium to phagocytose rod outer segments; this increases the concentration of large molecules and cellular debris in the SRF [2224].

During RRD repair, subretinal lavage may effectively lower oncotic pressure by removing most of the cellular debris and large molecules from the subretinal space. In this series, we demonstrated that subretinal lavage was feasible and safe. Also, transscleral drainage during vitrectomy allows remote SRF to be evacuated without creating an additional, often inferior, retinal break. This study improves our understanding of the pathogenesis of this relatively common condition and provides clues for improving surgical outcome. Further development of surgical technique is necessary. Lavage should preferably be isovolemic. A prototype of a dual bore cannula system to be used in non-vitrectomizing RRD repair is under development. This is particularly important since patients prone to develop PSF are mostly non-presbyopic and therefore cataract formation as a complication of $\mathrm{RRD}$ repair should be avoided.

\section{References}

1. Kaga T, Fonseca RA, Dantas MA, Yannuzzi LA, Spaide RF (2001) Optica coherence tomography of bleb-like subretinal lesions after retinal reattachment surgery. Am J Ophthalmol 132: 120-121.

2. Hagimura N, lida T, Suto K, Kishi S (2002) Persistent foveal retinal detachment after successful rhegmatogenous retinal detachment surgery. Am J Ophthalmol 133: 516-520.

3. Wolfensberger TJ, Gonvers M (2002) Optical coherence tomography in the evaluation of incomplete visual acuity recovery after macula-off retinal detachments. Graefes Arch Clin Exp Ophthalmol 240: 85-89.

4. Baba T, Hirose A, Moriyama M, Mochizuki M (2004) Tomographic image and visual recovery of acute macula-off rhegmatogenous retinal detachments. Graefes Arch Clin Exp Ophthalmol 242: 576-581.

5. Wolfensberger TJ (2004) Foveal reattachment after macula-off rhegmatogenous retinal detachments occurs faster after vitrectomy than after buckle surgery. Ophthalmology 111: 1340-1343.

6. Benson SE, Schlottmann PG, Bunce C, Xing W, Charteris DG (2006) Optical coherence tomography analysis of the macula after vitrectomy surgery for retinal detachments. Ophthalmology 113: 1179-1183.

7. Benson SE, Schlottmann PG, Bunce C, Xing W, Charteris DG (2007) Optical coherence tomography analysis of the macula after scleral buckling surgery for retinal detachments. Ophthalmology 114: 108-112.

8. Schwartz SG, Kuhl DP, McPherson AR, Holz ER, Mieler WF (2002) Twentyyear follow-up for scleral buckling. Arch Ophthalmol 120: 325-329.
9. Veckeneer M, Van Aken E, Cornelissen R, Derycke L (2008) Persistent Foveal Blebs: Are They Related to the Schwartz-Matsuo Syndrome? ARVO Meeting Abstracts 49: 4704.

10. McCulloch P, Altman DG, Campbell WB, Flum DR, Glasziou P, et al. (2009) No surgical innovation without evaluation: the IDEAL recommendations. Lance 374: 1105-1112.

11. Veckeneer M, Wong D (2009) Visualising vitreous through modified transscleral illumination by maximising the Tyndall effect. Br J Ophthalmol 93: 268270.

12. Heimann $H$, Bartz-Schmidt KU, Bornfeld N, Weiss C, Hilgers RD, et al (2007) Scleral buckling versus primary vitrectomy in rhegmatogenous retinal detachment: a prospective randomized multicenter clinical study. Ophthalmology 114: 2142-2154.

13. Salicone A, Smiddy WE, Venkatraman A, Feuer W (2006) Visual recovery after scleral buckling procedure for retinal detachment. Ophthalmology 113: 1734 1742.

14. Brazitikos PD, Androudi S, Christen WG, Stangos T (2005) Primary pars plana vitrectomy versus scleral buckle surgery for the treatment of pseudophakic retinal detachment: a randomized clinical trial. Retina 25: 957-964.

15. Mendrinos E, Dang-Burgener NP, Stangos AN, Sommerhalder J, Pournaras CJ (2008) Primary vitrectomy without scleral buckling for pseudophakic rhegmatogenous retinal detachment. Am J Ophthalmol 145: 1063-1070.

16. Seo JH, Woo SJ, Park KH, Yu YS, Chung H (2008) Influence of persistent submacular fluid on visual outcome after successful scleral buckle surgery for macula-off retinal detachment. Am J Ophthalmol 145: 915-922.

17. Gilbert GM, Owens SL, Smith PD, Fine SL (1984) Long-term follow-up of central serous chorioretinopathy. Br J Ophthalmol 68: 815-820.

18. Tsuboi S, Pederson JE (1988) Volume flow across the isolated retinal pigment epithelium of cynomologus monkey eyes. Invest Ophthalmol Vis Sci 29: 1652 1655.

19. Abouzeid H, Becker K, Holz FG, Wolfensberger TJ (2009) Submacular fluid after encircling buckle surgery for inferior macula-off retinal detachment in young patients. Acta Ophthalmol 87: 96-99.

20. Edmund J (1968) Analysis of subretinal fluid. Acta Ophthalmologica 46:1184 1193.

21. Takeuchi A, Kricorian G, Marmor MF (1996) When vitreous enters the subretina space. Implications for subretinal fluid protein. Retina 16: 426-430.

22. Immel J, Negi A, Marmor MF (1986) Acute changes in RPE apical morphology after retinal detachment in rabbit. A SEM study. Invest Ophthalmol Vis Sci 27 1770-1776.

23. Quintyn JC, Brasseur G (2004) Subretinal fluid in primary rhegmatogenous retinal detachment: physiopathology and composition. Surv Ophthalmol 49: 96-108.

24. Toti P, Morocutti A, Sforzi C, De Santi MM, Catella AM (1991) The subretinal fluid in retinal detachment. A cytologic study. Doc Ophthalmol 77: 39-46. 